\title{
Urología antigua en Galicia
}

\author{
L.A. Fariña Pérez \\ Servicio de Urología. Centro Médico POVISA, Vigo
}

Actas Urol Esp 2005; 29 (2): 121-130

\section{RESUMEN}

UROLOGÍA ANTIGUA EN GALICIA

Introducción: Aunque la Urología es una rama reciente de la ciencia y práctica médica, no resulta contradictorio hablar de Urología antigua, como una reflexión sobre las formas de enfermar y de tratar en épocas pasadas las enfermedades genitourinarias, padecimientos que son reconocibles al compararlos con las actuales. En este trabajo se tiene presente la visión de la historia de la medicina desde el punto de vista social y se procura dejar a un lado la pretensión de crear un pasado mítico para la urología o de subestimar las prácticas antiguas por irracionales o acientíficas.

Material y Método: Revisión basada en fuentes primarias y sobre todo secundarias, referidas a la medicina y cirugía de las vías urinarias y genitales en Galicia y en la península ibérica, durante la época antigua y medieval y hasta la aparición de la medicina anatomo-clínica en el siglo XVIII.

Resultado: La peregrinación por el Camino de Santiago hizo surgir en Galicia centros de gran importancia para la atención a los enfermos, como el Monasterio de San Martín Pinario y el Hospital de los Reyes Católicos, en los que se siguen las pautas tradicionales en Europa, de cuidados y uso de plantas medicinales. La medicina y cirugía antiguas de las vías urinarias y genitales tiene en Galicia personajes de gran interés, como el doctor Romano, que fue instructor de médicos gallegos en el sondaje y la dilatación uretral, e importantes pensadores como el médico filósofo Francisco Sánchez y los frailes ilustrados Sarmiento y Feijóo, quienes sin ser médicos, pueden ser considerados como precursores de la medicina basada en pruebas.

Comentarios: La importancia que en su tiempo tuvieron los hospitales gallegos y las obras de varios personajes relacionados con Galicia, ocupan un lugar destacado en la historia antigua de la cirugía de las vías urinarias y genitales.

Palabras clave: Historia de la cirugía. Historia de la medicina.

\section{ABSTRACT}

ANCIENT UROLOGY IN GALICIA (SPAIN)

Introduction: Although Urology is a recent branch of medical science and practice, it is not a contradiction to talk about ancient Urology, as a reflection on the ways of getting sick and treating genitourinary diseases in past times, sufferings that are recognized if compared to the present-day ones. This paper takes into account the social view of the history of medicine and tries to reject either the intention to create a mythic past for urology or to underestimate the ancient practices because of irrational or not scientific.

Material y Method: Review based on primary and secondary sources, with reference to the medicine and surgery of the urinary tract and genitalia in Galicia (Spain) and the Iberian peninsula, from the ancient and medieval periods, to the appearance of modern, anatomo-clinic medicine in the XVIIIth century.

Results: Because of the pilgrimage through the Way of Saint James to Santiago de Compostela, many centers of great importance for the attention of sick people rose up in Galicia, like the Monastery of Saint Martin Pinario and the Hospital of the Catholic Kings, where traditional European standars of patient care and use of medicinal plants were followed in. Ancient medicine and surgery of the urinary tract and genitalia had in Galicia, characters of great interest, such as doctor Romano, instructor of Galician practitioners in catheterisation and urethral dilatation, and important thinkers such as the physician- philosopher Francisco Sanchez and the erudite monks Sarmiento and Feijoo, whom, without being doctors, may be considered as pioneers of modern evidenced - based medicine.

Comments: The importance that the Galician hospitals had in past times and the works of several characters related to Galician country, had a distinguished place in the ancient history of the medicine and surgery of the urinary tract and genitalia.

Keywords: History of urology. History of medicine. 
$\mathrm{L}^{2}$ as nuevas corrientes de historiografía médica, surgidas en los últimos veinticinco años, han cambiado la visión bastante simplista que se tenía del progreso de la medicina y del nacimiento y desarrollo de las especialidades médicas, una visión basada en la épica de las conquistas médicas y en el predominio de las biografías de personajes supuestamente pioneros luchando contra el entorno y las creencias de su tiempo. Ahora se trata, más bien, de situar a la medicina y la cirugía en la corriente histórica general, relacionándola con la estructura social y las influencias políticas, culturales y económicas de cada momento y lugar $^{1-2}$ Los modernos profesionales de la historia de la medicina suelen mostrar, por este motivo, poco interés por la historia de las especialidades médicas tal como ha sido contada frecuentemente, -trazando una línea de continuidad entre un pasado lleno de ignorancia y sufrimiento y un presente de ciencia y de conocimiento-, pues se generan muchos errores históricos cuando se busca una explicación del pasado basada en el desarrollo lógico de los hechos, y dejan de considerarse otras explicaciones menos racionales y más complejas .

Quienes trabajan en esta nueva visión, eminentemente social, de la historia de la medicina, sugieren mirar con otros ojos algunas prácticas antiguas calificadas como irracionales o acientíficas, de manera que podamos llegar a apreciar su coherencia y sabiduría en el contexto social y la época en que tuvieron lugar. También nos recuerdan lo injustificado de hacer juicios sobre la medicina antigua en función de las prácticas que se tienen hoy en el mundo occidental, pues, por ejemplo, estudios recientes han mostrado cómo la medicina medieval galénica y árabe ofrecían ya una red de asistencia médica y medios para abordar el mundo de la enfermedad de su tiempo y satisfacer el deseo de salud de una parte de la población ${ }^{3}$.

Por otra parte, carece de toda base científica y racional, la tentación de crearse un pasado glorioso, presente en las historiografías de la mayor parte de las especialidades médicas y quirúrgicas, pues es sabido que la aparición de las especialidades desde los troncos comunes de la medicina y la cirugía es un fenómeno reciente. En el caso de la Urología, es consecuencia de la coinci- dencia de circunstancias como la especialización en el diagnóstico y tratamiento de las estrecheces uretrales venéreas, el invento de instrumentos endoscópicos, el hallazgo de técnicas de laboratorio para el análisis de la orina y de la función renal, y, más recientemente, el desarrollo de exámenes radiológicos del tramo urinario con contrastes.

Aunque la Urología es una rama reciente de la ciencia y práctica médica, hablar de Urología antigua no resulta contradictorio, si aclaramos que se trata de reflexionar sobre las formas de enfermar y de tratar en épocas pasadas las enfermedades genitourinarias, padecimientos que son reconocibles al compararlos con la actuales, reconociendo que esta reflexión puede tener el sesgo de una visión centrada en la especialidad tal como es ahora, y los sesgos consecuencia del uso preferente de fuentes bibliográficas secundarias, por tanto con las limitaciones y riesgos de una metodología alejada de la propia de la Historia de la Ciencia ${ }^{4-5}$.

\section{UROLOGÍA MEDIEVAL EN GALICIA}

Galicia siempre fue una de las regiones más densamente pobladas de la península ibérica. $\mathrm{Su}$ clima es húmedo, pero el invierno es benigno y no hay excesivos contrastes térmicos, por lo que muchos pueblos antiguos se asentaron en su territorio. Pocas evidencias existen de la medicina en los primeros siglos de la era cristiana, durante los que se mantuvo la influencia de los habitantes de los castros. La dominación romana fue superficial en Galicia, aunque dejó importantes señas arquitectónicas y de organización social, y, en el plano médico de interés urológico, la medicina y cirugía bélica, el uso terapéutico de la orina, de las plantas y hierbas y de las aguas medicinales, que ya mencionaba Plinio (baños en Orense o Lugo), perviviendo en nuestros días la importancia de estos últimos ${ }^{6}$. Después de suevos y visigodos, los musulmanes, con una organización social tan avanzada ${ }^{7}$, apenas permanecieron 30 años en Galicia, que se mantuvo, por tanto, bastante al margen de la influencia cultural y política de la Reconquista. El "descubrimiento" de la tumba del apóstol Santiago en el primer tercio del siglo IX y su enorme repercusión en todo el mundo cristiano, fue unos de los 
hitos más importantes de la historia de España, y puso en el camino a Santiago de Compostela a peregrinos de todas las procedencias. Se desarrolló una red viaria que desde toda Europa se dirigía hasta Compostela, a través de distintos "caminos de Santiago", que se fueron jalonando de obras públicas y lugares de culto al Apóstol. También surgió un sistema asistencial que estaba en manos de órdenes religiosas, las cuales crearon monasterios, albergues y hospitales de ayuda a los peregrinos sanos y enfermos y a los pobre, en los lugares mejor situados de esas transitadas rutas medievales. En tierras gallegas, Cebreiro, Portomarín, encomendadas a los Templarios desde 1142 hasta la supresión de esta orden en el siglo XIV, y luego sustituidos por los Hermanos Hospitalarios de San Juan de Jerusalén, que se instalaron en Quiroga, Rivadavia y otras partes; los benedictinos en Samos, Celanova y Santiago (San Martín Pinario y San Paio), y los atendidos por monjes cistercienses en Sobrado, Oia y Oseira ${ }^{8-9}$. Los grandes monasterios eran casas de enfermos, peregrinos $\mathrm{y}$ viajeros tanto como de monjes, y éstos eran depositarios del saber médico, una situación generalizada en Europa. En los siglos precedentes la medicina había ido adquiriendo su carácter de ciencia curativa basada en la observación de la eficacia de los medicamentos, plantas, flores y minerales y hubo una separación lenta entre las figuras del médico, cirujano y boticario, finalmente sancionada en leyes que prohibían expresamente la colusión de estos oficios, para mayor seguridad de los enfermos. Estas tres figuras suelen estar presentes en los grandes monasterios gallegos, que tienen un carácter asistencial y no sólo de albergue. Los monjes conocían las propiedades de las hierbas -tónicas, depurativas, excitantes, balsámicas y sedantes o narcóticas-, fabricaban todo tipo de bálsamos, jarabes, ungüentos, vinos y licores, y contrataban los cuidados de un médico y de un cirujano, bien en ocasiones uno de los monjes tenía esta formación, bien era enviado a estudiar para atender las necesidades de los enclaustrados y de las poblaciones y comarcas vecinas. Reconocen y tratan el cólico de flanco, los síntomas miccionales, la retención de orina y las enfermedades de los genitales externos ${ }^{9}$. Las enfermedades venéreas eran entonces tan comunes, que se crearon hospitales monográficos dedicados a atender y controlar su transmisión, primeros centros especializados, cuya misión era aislar: diversas epidemias de "bubas" movieron al arzobispo Francisco Blanco a fundar en Santiago en 1577 el Hospital de San Roque, dedicado al mal gálico y a otros contagiosos. Dispuso de médico, cirujano y sangrador, y llegó a atender hasta comienzos del siglo XX a unos 600 pacientes al año, procedentes de Galicia y territorios limítrofes, afectos de supuestos males venéreos, que eran tratados con preparados de mercurio y tisanas de palo santo, sándalo y zarzaparrilla ${ }^{10}$. La lepra era también un mal endémico, y en las afueras de muchas ciudades existían leproserías para recoger a los enfermos elefantiásicos o "gafos", lo que seguramente incluía varias enfermedades de la piel y de los genitales, algunas de las cuales se conocía que eran contagiosas. La Orden de San Lázaro protegía a estos enfermos y en Santiago se fundó en 1187 el Hospital de San Lázaro para los canónigos leprosos de la Colegiata de Sar y para otros leprosos admitidos por el prior de esta Colegiata. En Monforte, se conserva la Capilla de San Lázaro, que fue una ermita anexa a un Hospital dedicado al mismo Santo ${ }^{11}$.

\section{UROLOGÍA EN GALICIA BAJ O LOS REYES CATÓLICOS Y LA CASA DE AUSTRIA}

A partir del siglo XI, después de que en la guerra con los musulmanes se afianza la frontera del Tajo y cuando la ruta de Santiago tiene una relevancia definitiva, comienza a repoblarse Galicia: en el siglo XVI tenía 900.000 habitantes, la vida se concentró en el litoral, con actividad marinera favorecida por sus puertos naturales, y Santiago es la ciudad más poblada y el centro político y espiritual. Antes de los Reyes Católicos, se repartían el poder Galicia unos cuantos nobles (señores de Andrade, Ulloa, Trastamara, Sotomayor), junto con los abades de los monasterios y el arzobispo de Santiago, estando los campesinos en la más completa miseria. En el siglo XIV y XV las luchas sociales fueron constantes entre señores y de los campesinos contra nobles y obispos: el levantamiento Irmandiño de 1465 expulsó a los señores de Andrade y Sotomayor y al arzobispo 
de Fonseca, pero fue al final derrotado y de nuevo sometido. Finalmente, los Reyes Católicos anularon el poder de los nobles, si bien no hubo ninguna mejora para los campesinos. Por entonces el mayor centro asistencial era en Santiago el Monasterio de San Martín Pinario (Fig. 1), con una gran botica, exponente principal del movimiento farmacológico basado en plantas que había en los monasterios, similar al que tuvo lugar en toda Europa hasta el siglo XVIII ${ }^{12}$. Aún en 1705 se ampliaron las dependencias de su botica y del jardín botánico, dedicando espacio para el cultivo de plantas curativas traídas de otros lugares por indicación de Fray Antonio, cirujano del monasterio, muy diestro en hacer preparados eficaces contra el mal de retención de orina, entre otros ${ }^{13}$. Para los sintomas miccionales y la retención de orina se usaban las plantas con efecto diurético y analgésico, como la manzanilla, la escila (también para los cólicos de riñón), el ajo, el saúco y el acónico, esta última conocida por su toxicidad ${ }^{13-14}$.

El Hospital Real o de los Reyes Católicos (Fig. 2) fue fundado en 1492, después de la conquista de Granada, como ofrenda de agradecimiento de los reyes Fernando e Isabel, devotos del Apóstol y peregrinos a Compostela en varias ocasiones.

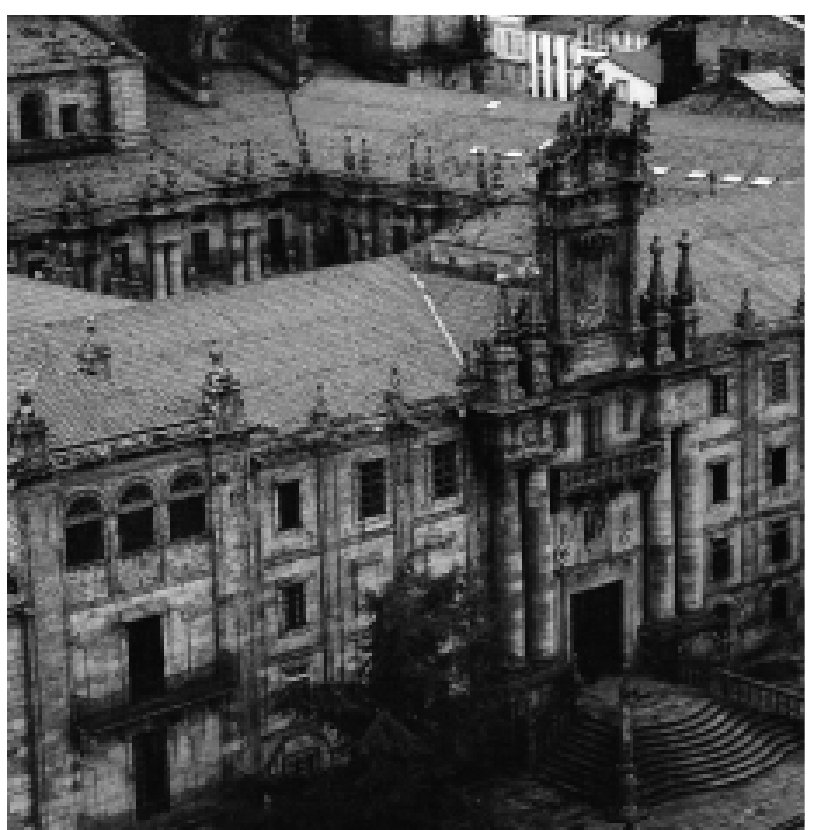

FIGURA 1. Monasterio de San Martín Pinario, el mayor centro asistencial en el Santiago medieval, provisto de una gran botica de plantas medicinales.
Durante varios siglos fue uno de los mejores establecimientos de su tipo en el mundo y dio refugio $\mathrm{y}$ atención médica y quirúrgica a peregrinos $\mathrm{y}$ pobres y más modernamente fue, como Hospital Clínico, el principal centro de la medicina y la cirugía en Galicia, hasta que en 1954 pasó a ser un hotel. Aunque su administrador recordaba frecuentemente a las autoridades civiles su misión primitiva de servicio a los peregrinos, no pudo evitar la obligación de la asistencia colectiva a la ciudad de Santiago y las comarcas de Galicia. Las Reales Constituciones de Carlos V disponían una consulta ambulatoria en la Puerta Real del Hospital, en la que se hacía examen de la orina:

“...que acavado la visita de la mañana, estén (los médicos) un quarto de hora a la puerta del Hospital, viendo aguas, $y$ aconsejando a algunos vecinos de la Ciudad pobres, ... o si se requiere sangría, vaya el barbero a facerla de valde."

Durante el reinado de los Austria hubo un estancamiento significativo en la medicina y la

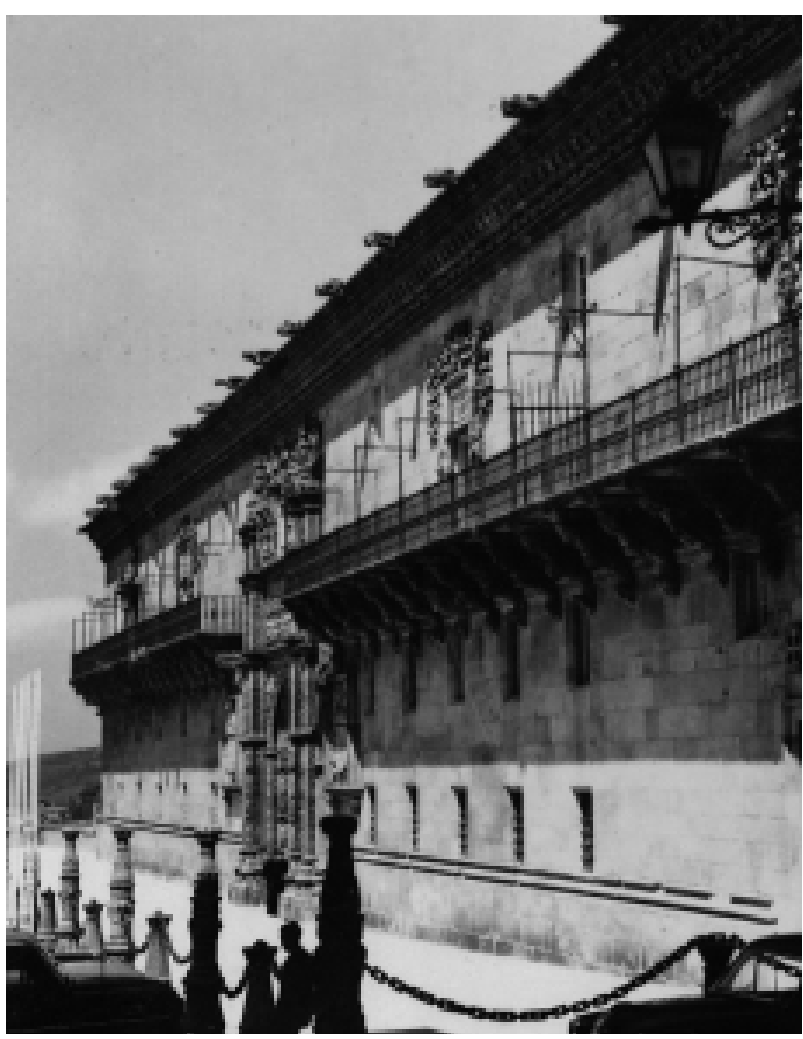

FIGURA 2. Hospital Real de los Reyes Católicos, que fue durante muchos siglos uno de los mejores establecimientos del mundo, y sede de la Escuela médica gallega de finales del siglo $\mathrm{XIX}$ y comienzo del $\mathrm{XX}$. 
cirugía. Las Constituciones de Carlos V hacían al médico responsable de la uroscopia, mientras que el cirujano tenía una misión subordinada a la de aquél:

"al tiempo de la visitación, mandamos que el médico sea obligado a mirar las aguas de cada enfermo, y detenerse con cada uno algún espacio para informarse dél largamente..., y mire y tiente los pulsos, toque y tiente las partes del cuerpo que convenga..."

"que haya en el dicho nuestro Hospital un cirujano examinado, el mejor que se pudiere haber. El cual visite los enfermos de su oficio dos veces al dia, y mas si fuere menester, ...y cuando ocurriere algún accidente, llamará al médico de casa. Y con su acuerdo, y no sin él, entienda en la cura..."15.

Esta separación entre las actividades de médico y cirujano era muy rigurosa. Todavía una disposición promulgada en 1721 nos da una idea de la escasa consideración que el trabajo del cirujano tenía, al denunciar “...la peligrosa introducción $y$ delito de los cirujanos de galeras de que han ordenado y dado por sí mismos sin conocimiento de médico, los dos remedios más principales y de consecuencias de vida, de sangrias y purgas...". En la mejor época del imperio naval español, el cirujano de un buque debía alojarse con el resto de la tripulación y sólo estaba mejor considerado que los remeros. Estaba también obligado a comprar los instrumentos necesarios para ejercer su oficio, básicamente para hacer amputaciones, trepanaciones y usar el cauterio. Entre este instrumental se incluían cuatro algalias graduadas, para el sondaje uretral ${ }^{16}$.

Casi trescientos años después de la fundación del Hospital Real, en las Constituciones de Carlos IV de 1804, las cosas apenas habían cambiado:

“(el médico)... hará al enfermo las preguntas que tenga por necesarias; reconocerá sus aguas; lo pulsará; le tentará las partes del cuerpo que convenga..."

El Hospital Real tenía una excelente botica y bien provista huerta, y siempre dispuso de uno o más cirujanos, si bien no es probable que hubieran realizado intervenciones urológicas de envergadura, pues entre otras cosas no disponía de un anfiteatro anatómico. En los siglos XVI y XVII, hasta que se regularon los estudios de medicina y cirugía, era muy escaso el número de profesio- nales. Prácticos de escasa formación pero que llegaron a tener buena experiencia en el tratamiento de enfermedades internas y externas, sin llegar a completar estudios, tenían consideración social y recibían el nombre de maestro o maestre. Así por ejemplo, Juan de Zaldívar en la ciudad de Santiago ejercía "el oficio de cirugía y curar llagas viejas y humores malos y mal de bubas $y$ dar agua del palo..." (refiriéndose a la infusión de palo santo para tratar la sífilis), todo ello "en vista faz e paz de los médicos e cirujanos de esta cibdad e de los del Gran Hospital Real, y con su aprobación..." Una de las crónicas de sus tratamientos, recuperada por Pérez Constanti ${ }^{17}$, relata una posible gonococia complicada con abscesos y fístulas perineales que finalmente curó con el tratamiento instaurado:

“...Roy Fernández regidor desta ciudad, (...) estaba abierto y con ciertos agujeros en la rabadilla por do orinaba y con él abían andado todos los médicos de la dha ciudad y con ellos se gastaron más de seiscientos ducados y al cabo lo dexaron abierto y desahuciado y peor, hasta que el dho maestre Juan vino a entender en vista de los dhos médicos, en la dha cura y le dio ciertas medicinas (...) y con ungüentos (...) le hizo volver en salud natural como hay día está muy bueno y sano y los agujeros cerrados."

En 1565 llegó a Galicia el doctor Romano, que ya tenía una fama reconocida, en una misión oficial que tenía por objeto enseñar a otros médicos su técnica de sondaje con candelillas en los estrechados de uretra y los que sufrían de retención de orina. Romano, llamado Alfonso o Diego Díaz, es nombrado en los escritos de Andrés Laguna y de Francisco Díaz, por ser uno de los primeros en conocer la dilatación uretral con candelillas. El 17 de Noviembre se presentó en el Concejo de Santiago con una resolución de las Cortes en la que se leía:

Muy magníficos Señores. El Reino estando junto en Cortes tomó cierto asiento y concierto con el Doctor Romano, cirujano, para que curase en estos Reinos la enfermedad de retención de orina y avezase el arte e cura della a los médicos e cirujanos que los Ayuntamientos de las cibdades y villas le declarasen, sin les llevar por ello cosa alguna, y que a los pobres que ubiese de la dha enfermedad les curase de balde y a los otros les 
llevase una moderada paga (...), y (...) el Reino acordó que el dicho Doctor Romano visitase el Reino de Galicia e curase en él conforme a su instrucción los enfermos que ubiese de la dicha enfermedad (...). El qual va a lo cumplir; y pues la obra es tan buena, vuestras mercedes provean que el primero o segundo dia que llegare le nombren los médicos e ciruxanos que ha de abezar la dha arte e cura e que se publique para que los que tuvieren la dicha enfermedad en su comarca y partido, se bengan a curar con él conforme a la dicha instrucción, y dársele testimonio de las curas que hiciese e de los médicos y cirujanos que abezare (...) y porque somos ynformados que demás de los médicos que hay en esa cibdad, ay en algunas villas $y$ lugares de su obispado otros muchos, mandarán vras. mds. abisalles (...), para que todos los que quisieren aprender lo puedan hazer (...).

Se pregonó la resolución en La Coruña, Betanzos, Noya y otros lugares de la entonces provincia de Santiago, y aprendieron el arte prácticos de formación diversa, que hicieron juramento de tratar de manera gratuita a los pobres y de enseñar a otros lo que habian aprendido. Después de dos meses y medio de estancia en Santiago, recibió el doctor Romano 75 reales, a razón de un real por día, y partió para otras ciudades $^{18}$.

En el siglo XVI y parte del XVII fueron de uso frecuente en Galicia, los contratos que vinculaban a las partes para la provisión de atención médica o quirúrgica. Una parte del salario solía pagarse por adelantado y el resto si fuera o no exitosa la asistencia, dependiendo de las particularidades del contrato. Durante el tiempo que durara el tratamiento, el paciente abonaba el alojamiento y manutención del práctico, que tantas veces actuaba como ambulante. En no pocas ocasiones, en caso de disputa o de fallecimiento del paciente, se pedía a terceros que tasasen el trabajo realizado ${ }^{19}$.

Aunque nacido probablemente en Tuy (Pontevedra), el médico filósofo Francisco Sánchez (1551-1623) (Fig. 3), no tuvo relación profesional con Galicia, pero llegó a ser una de las más eminentes figuras médicas de su tiempo desde su cátedra de Medicina en Montpellier y como maestro de cirujanos en Toulouse. Conocido como "el Escéptico" por su pensamien-

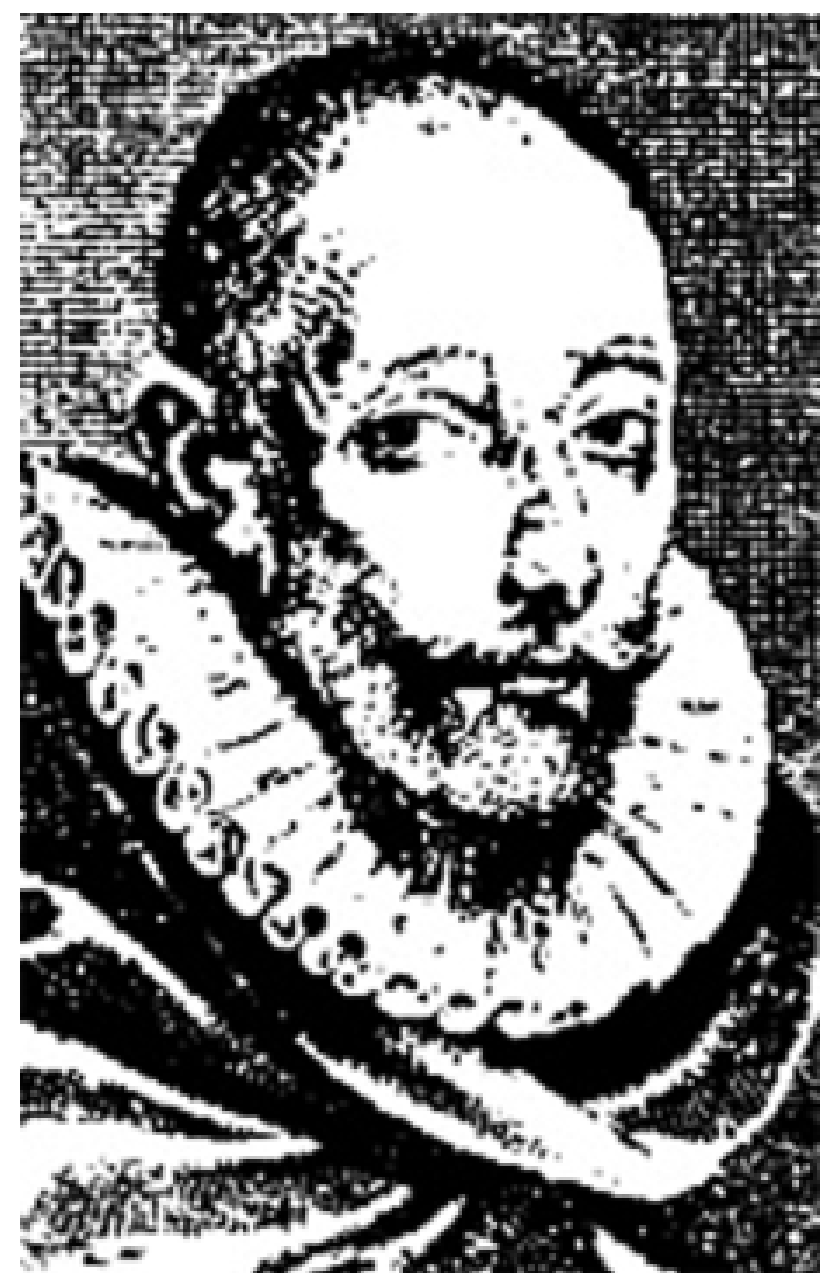

FIGURA 3. Francisco Sánchez, filósofo escéptico y ciruja no en Montpellier y Toulouse.

to recogido en el libro de 1581 "Tratado de la muy noble y primera universal ciencia, que nada se sabe", es el precursor de la duda metódica preconizada por René Descartes, que tanto contribuyó al pensamiento científico moderno. Entre sus escritos se encuentran textos relativos al conocimiento de la época sobre temas relacionados con la hoy llamada andrología (impotencia sexual y generadora) $^{20}$.

\section{UROLOGÍA EN GALICIA BAJ O LA CASA DE BORBÓN}

Hacia finales del siglo XVII Galicia pasaba del millón de habitantes, la mayor parte siervos y en la costa marineros tan pobres "que tenían que comer ostras" ${ }^{21}$. El padre Sarmiento a principios del siglo XVIII habla de la necesidad de "la resurrección de este muerto país" y de la infinidad de 
pobres infelices que son esclavos de la tierra. Las tierras eran de las iglesias y de los nobles, y todas las rentas iban a parar a Castilla. A lo largo de este siglo XVIII hubo años en que las hambres fueron terribles, y se inició la emigración masiva a Castilla para la siega, a Madrid y a Lisboa para los trabajos manuales. En el período del Imperio español en América y en el Atlántico habían crecido las ciudades costeras como Pontevedra, Vigo, Bayona, La Coruña y Ferrol, etapa que se interrumpió con el desastre de la "Armada Invencible" y la necesidad de que los envíos a Flandes, antes por mar, pasasen ahora por Italia. En este desastre naval el pequeño Hospital de San Andrés del gremio de mareantes de La Coruña tuvo un papel principal en la atención a los marinos que llegaban diezmados por las enfermedades y la falta de alimentos a bordo.

A lo largo de la mayor parte del siglo XVIII el Hospital Real de Santiago estuvo saturado de enfermos afectos de problemas epidémicos y carenciales, con una elevadísima mortalidad. En un acta de 1769 se afirmaba: "...llegan diariamente crecido número, unos que alcanzan escasamente la extremaunción; otros del todo deplorables; y no pocos que los traen enteramente muertos, (...) habiendo entrado desde primero de enero más de dos mil pobres, $y$ muerto en este tiempo quinientos y dos...". Durante prolongados períodos, los enfermos incurables y los moribundos eran sacados del Hospital y abandonados a la caridad pública de las calles. El retraso con que se inició en España la enseñanza clínica y anatomopatológica que en Paris, Londres o Viena estaban cambiando la medicina, fue muy patente en Galicia, cuyo Colegio de Medicina de Santiago -con sede en el Colegio de Fonseca- no tuvo hasta 1755 su primera cátedra de Anatomía y Cirugía: Pedro Gómez de Bedoya, solicitó la plaza en un escrito en el que ponía como condición disponer de "teatro capaz para las disecciones, instrumentos para ellas, losa y disector, no ha de ser de mi cuenta el coste de ello". Finalmente se limitó a explicar sus lecciones de anatomía, al no disponer de ayudante para las disecciones. Por otra parte, el Hospital fue reticente a proporcionar cadáveres para el estudio y a que en su interior se realizaran autopsias $\mathrm{u}$ otro tipo de docencia dirigida a los estudiantes, situación que no cambia hasta que en
1799 se establece en él un Colegio de la Facultad Reunida médico-quirúrgica, después Colegio de Cirugia $^{22}$. Tampoco se aprovechó la breve estancia en Santiago del cirujano francés Juan Delgart, que había sido "cirujano demostrador en anatomía y operaciones de cirugía” en Paris y Bayona y que se instaló aquí después de haber acompañado a los ejércitos Borbones en la guerra de sucesión con los Austria por el trono de España (17011704). Alguno de estos cirujanos franceses tenía experiencia en la litotomía perineal.

En varias áreas de las ciencias se realizaron en Galicia importantes estudios en este período de la Ilustración ${ }^{23}$, un momento en que el analfabetismo, la religiosidad y la superstición son rasgos culturales predominantes en la mayoría de la población. Dos monjes benedictinos relacionados con Galicia destacan en estos años por sus textos sobre la medicina, si bien no la practicaron abiertamente, pero sí a través de sus recomendaciones, que tuvieron mucho predicamento: los frailes Sarmiento y Feijóo. Martín Sarmiento (16951771) (Fig. 4) llegó a tener profundos conocimientos en todas las ramas del saber, particularmente

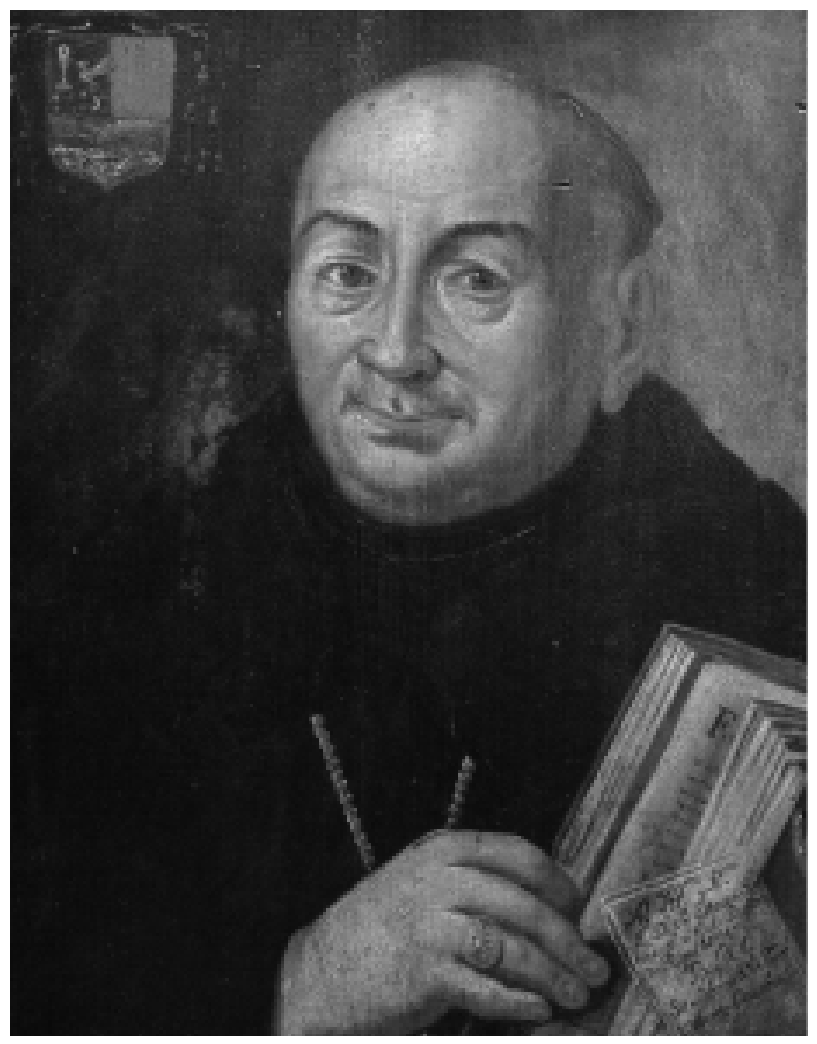

FIGURA 4. Fray Martín Sarmiento, óleo de M. Cousiño. 
en la botánica. En su Viaje a Galicia, de 1745, recoge los nombres de las hierbas del lugar, comparando el nombre vulgar y el uso que le daban los nativos, con el que aparecía en tratados como el Dioscórides comentado por Andrés Laguna y la Historia Natural de Plinio. Algunas, de uso en afecciones urinarias: así por ejemplo el fiuncho, diurético y aperitivo en infusión; la infusión de ramas y hojas del abedul eran en su opinión muy eficaces contra el mal de piedra y las enfermedades de la orina: observó que estas enfermedades eran relativamente raras en Galicia, y lo relacionó con la costumbre de comer en vasos y platos de madera de abedul. La xesta, diurética; la verbena para los males de hígado, bazo y riñones. El agua de cocer los granos de maíz, "es remedio eficaz contra la supresión de orina, ...por eso los americanos no padecen ni de piedra, ni de riñones, ni de orina" ${ }^{24}$. La planta conocida como carquesa o carqueixa (Fig. 5), la consideraba una panacea para todo tipo de traumatismos y enfermedades, incluido el cólico renal, y muchos médicos de su tiempo la usaron. Gaspar Casal, autor de Historia natural y médica del Principado de Asturias (1762), el más importante tratado de medicina en la España del siglo XVIII ${ }^{25}$, le visitó en su celda de Madrid para conocer las propiedades de esta planta. Benito Jerónimo Feijóo (16761764) (Fig. 6) ingresó en la orden benedictina de Samos-Lugo y tras estudiar en Lérez y Salamanca pasó al convento de San Vicente de Oviedo en 1709, del que fue abad, y en cuya Universidad obtuvo diversas cátedras. Después de pasar muchos años dedicado al estudio, publicó sus dos obras enciclopédicas, el Teatro crítico universal y las Cartas eruditas y curiosas, que tuvieron una enorme difusión en España y fueron traducidas a los principales idiomas europeos. Llegó a ser conocido en toda Europa, y recibía en su celda a discípulos de toda España. Sus obras, que recogen las novedades científicas y técnicas de la época, "abrieron las puertas de la razón” en España. Se enfrentó a tradiciones y abusos que hicieron de él un precursor del pensamiento ilustrado español, y criticó toda teoría avalada sólo por la tradición o la autoridad, aunque nunca cuestionó los dogmas de fe. Propugnó la reforma de los estudios médicos y de otras materias, proponiendo una terapéutica basada

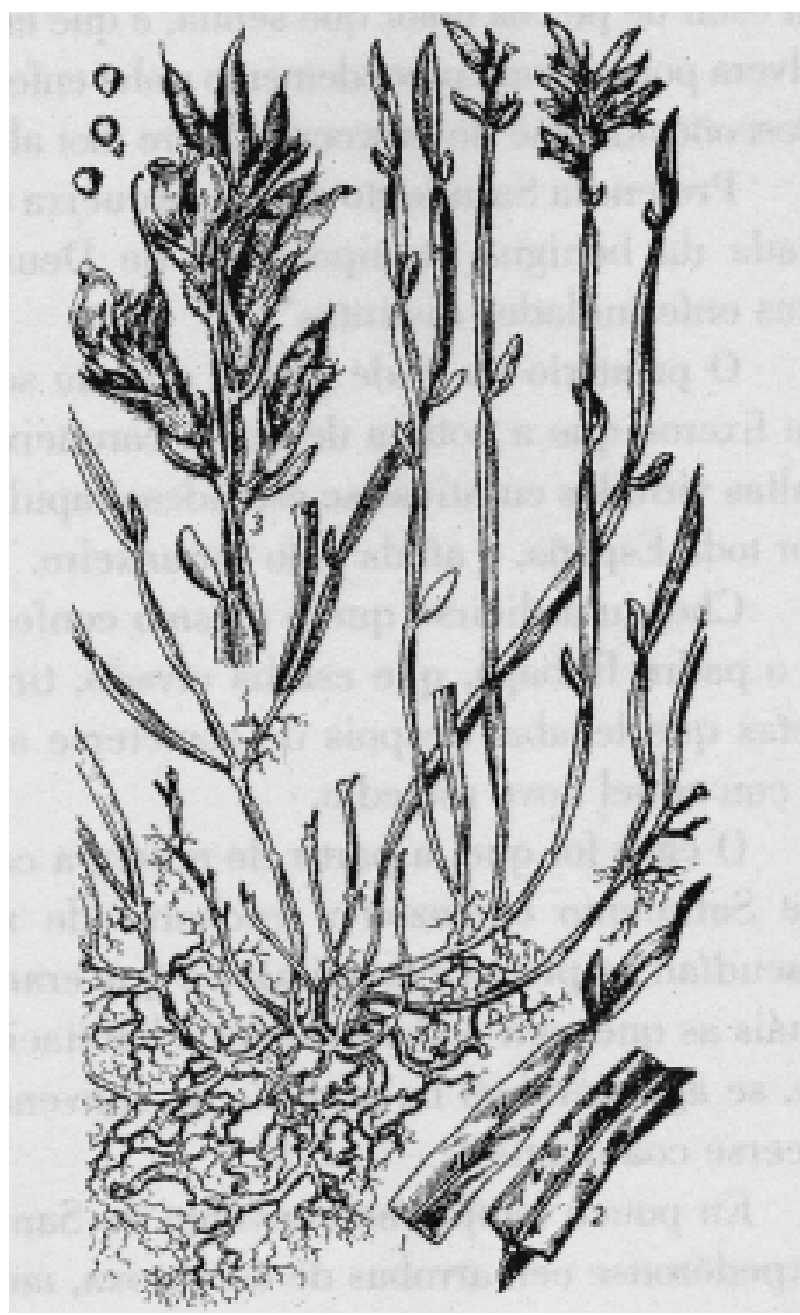

FIGURA 5. La planta carqueixa, considerada por Sarmiento y por muchos médicos de su tiempo una pana cea para todo tipo de traumatismos y enfermedades, incluido el cólico renal.

en el sentido común y criticando la que se aplicaba en su tiempo, llena de empirismo con su fundamento en los aforismos hipocráticos, las supersticiones y los medicamentos tradicionales sin un sustento experimental. Es por eso un auténtico precursor de la actual "medicina basada en pruebas".

Algunos monasterios como Samos y Meira en Lugo, y Oia en Pontevedra, fueron usados como hospitales de tropas durante la guerra de la Independencia con Francia. Posteriormente, las leyes de desamortización fueron la ruina de la mayor parte de los monasterios de Galicia, incluidos archivos y bibliotecas, pese a que se pretendió evitarlo con inventarios y valoraciones previas. 


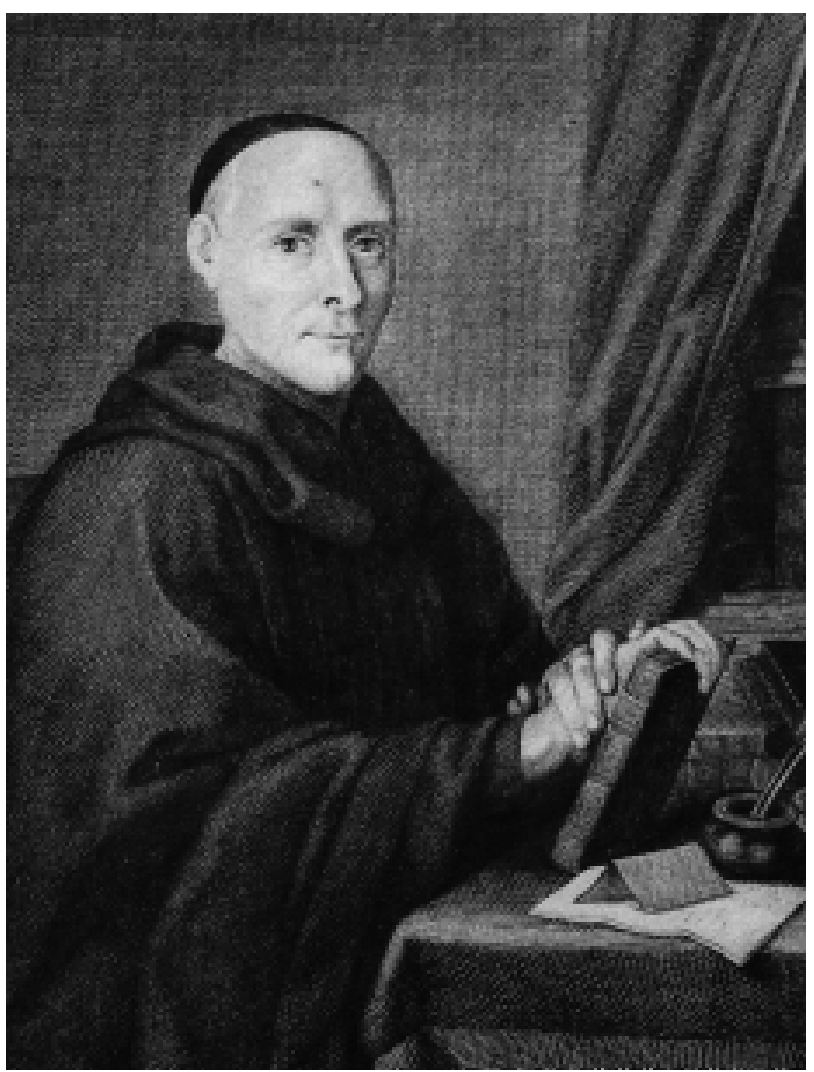

FIGURA 6. Fray Benito Jerónimo Feijoo, según un grabado de la época.

\section{UROLOGIA EN GALICIA EL SIGLO XIX: ORIGENES DE LA UROLOGIA MODERNA}

Los finales del siglo XVIII, es la época en la que se fija el nacimiento de la medicina moderna, surgida del pensamiento médico anatomoclínico de los hospitales de Paris, Viena y Londres, consecuencia a su vez del dominio de la anatomía logrado en los siglos anteriores, del más reciente de la anatomía patológica y de la sistematización de los cuadros clínicos. Este nuevo paradigma de la medicina, según el cual el estudio de un paciente requería la observación de sus órganos enfermos, se sustentó luego con la mejora de los hospitales, los progresos de la instrumentación y los conocimientos sobre la infección y la anestesia, y terminó superando la antigua tradición hipocrática y galénica, que en ese momento resultaba anquilosada y carente de respuestas para los numerosos problemas de salud de la nueva sociedad 26. Los cambios que experimenta la medicina y cirugía en España, se retrasaron significativamente, como consecuencia de la situación política y económica del país y el declive de su Imperio, pero la creación de los Reales Colegios de Cirujanos de Cádiz y los de Barcelona y Madrid, contribuyó de manera definitiva a la formación académica de médicos y cirujanos para la práctica militar y civil. También contribuyeron otros cambios que se sucedieron a continuación, como la creación de nuevas Facultades de Medicina y la fusión de los estudios de medicina y cirugía, lo que también tuvo lugar en Santiago. La población en Galicia era en este momento muy rural y dispersa, con el $80 \%$ localizada en núcleos de población de menos de 200 habitantes, por lo que la atención médica, con un número muy limitado de profesionales, tenía que ser muy insuficiente, pero a lo largo del siglo XIX en las ciudades más grandes como La Coruña, Santiago y Vigo se formaron núcleos burgueses que tuvieron cierta influencia en la España del período liberal, y en este periodo, la asistencia sanitaria pasará a ser prestada en Hospitales civiles y militares ${ }^{27-28}$.

A finales del siglo XIX y comienzos del XX, a medida que mejora la higiene de las ciudades y la alimentación de sus habitantes, van disminuyendo las enfermedades carenciales y transmisibles. Entonces necesitan más atención un grupo de enfermedades consuntivas conocidas como tisis, y otras enfermedades crónicas incurables; así como también la sífilis y la gonococia, que se atenderán en Centros donde comienza a practicarse la urología del tramo urinario inferior. En este período, los enfermos gallegos continuarán peregrinando a Santiago, pero esta vez para ser atendidos en un Hospital Real de los Reyes Católicos, en donde practican médicos y cirujanos que fueron pioneros e influyentes en España, un grupo tan notable y compacto que llegó a ser conocido como Escuela Médica Gallega. Este Hospital Real en 1901 tenía ya una de las mejores instalaciones de rayos $\mathrm{X}$ del país, $\mathrm{y}$ en él se iniciarán las intervenciones urológicas con anestesia y asepsia que suponen el comienzo de la Urología moderna en Galicia, comentado en un trabajo anterior $^{29}$. 


\section{REFERENCIAS}

1. Lindeman M. Medicina y sociedad en la Europa moderna (15001800). Siglo XXI de España, Madrid, 2001.

2. Marandola P, Musitelli S, Jallous H. Outline of a critical survey of urological historiography De Historia Urologiae Europeae. 1996;3,175-199.

3. García Ballester L. La búsqueda de la salud. Sanadores y enfermos en la España medieval. Península, Barcelona, 2001.

4. Kragh H. Introducción a la historia de la ciencia. Crítica, Barcelona, 1989.

5. Musitelli S, Jallous H, Marandola P. An introduction to methodological problems in the history of urology. De Historia Urologiae Europeae 1996;3, 137-173.

6. Leboreiro MA. A vida nos balnearios de Galicia. Ir Indo. Vigo, 1996.

7. García Ballester L. Historia social de la medicina en la España de los siglos XIII al XVI. Akal, Madrid, 1976.

8. Vázquez Gallego J. Los hospitales del camino francés de Galicia. Ediciós do Castro, A Coruña; 2001.

9. De Sa Bravo H. Boticas monacales y medicina naturista en Galicia. Everest, León 1983;32-39.

10. Carro Otero X. Cura de sifiliticos en Compostela. En: Materiais para unha historia da medicina galega. Xunta de Galicia, Santiago de Compostela; 1998;19-27.

11. Moure-Mariño L. El antiguo Hospital de San Lázaro y el de Sancti Spiritus En: Apuntes para la historia de Monforte de Lemos. Xunta de Galicia, Santiago de Compostela; 1997;51-54.

12. Girre L. Traditions et propriétés des plantes médicinales. Histoire de la pharmacopée. Privée, Paris, 1997.

13. De Sa Bravo H. op. cit., p 43.

14. Sanmartín Sarmiento JS. Monjes y boticarios. La farmacia del monasterio de San Martín Pinario. Nigra-Consorcio de Santiago, Vigo; 1997; 97-146.

15. García Guerra D. Constituciones, Organización y Gobierno. En: El Hospital Real de Santiago (1499-1804). Fundación Barrié de la Maza, La Coruña; 1983;137-233.

16. Novo Lopez JE. Evolución histórica de la medicina naval. Universidad Complutense, Madrid; 1989;93.
17. Pérez Constanti P. Especialidades médicas en el siglo XVI en Galicia . En: Notas viejas galicianas, tomo 2. Imprenta de los Sindicatos Católicos, Vigo 1925;167-175.

18. Pérez Constanti P. Los médicos gallegos aprendiendo a curar el mal de retención de orina. En: op. cit,161-165.

19. Pérez Constanti P. Los contratos de asistencia médico-quirúrgica. En: op. cit.,153-159.

20. Alvarez Blázquez D. Francisco Sánchez El Escéptico. Imprenta Sago, Vigo 1964.

21. Losada B. Galicia. Taber, Barcelona, 1969;111.

22. García Guerra D. El Hospital Real y la renovación de la enseñanza de la medicina. En: op. cit.381-440.

23. AA.VV. Os Ilustrados galegos: Reforma e tradición na Galicia do Antigo Réxime. Deputación Provincial da Coruña. A Coruña, 1996.

24. Sarmiento M. Viaje a Galicia (1745). Museo de Pontevedra, Pontevedra, 1975; 151

25. Casal G. Historia natural y médica del Principado de Asturias 1762. Edición facsímil. Principado de Asturias, Oviedo 1988.

26. Meyer PH, Triadou P. Leçons d’histoire de la pensée médicale. Sciences humaines et sociales en médicine. Odile Jacob, Paris, 1996.

27. Barreiro Mallón B, Rey Castelao O. Pobres, peregrinos y enfermos: La red asistencial gallega en el Antiguo Régimen. NigraConsorcio de Santiago, Vigo;1997;87-111.

28. Fernández Carlos M. Antiguos hospitales de La Coruña. Vía Láctea. La Coruña, 1995;106-132.

29. Fariña LA. La urología en Galicia y Asturias. En: Maganto Pavón E, Pérez Albacete M. Historia biográfica y bibliográfica de la urología española en el siglo XX. Edicomplet, Madrid, 2002;203218.

\section{L.A. Fariña}

Centro Médico POVISA,

Calle Salamanca, 5

36211. Vigo 